Rheumapatienten ist generell zu empfehlen, sich bei Auftreten von Augenbeschwerden wie Fremdkörpergefühl, Lichtempfindlichkeit, Schlieren-Sehen, Sehverminderung oder Organgefühl (Spüren des Auges) rasch an einen Augenarzt zu wenden.

Quelle: Pressekonferenz,NNeuigkeiten aus der Rheumatologie", Wien, 11. Oktober 2016

\author{
Literatur \\ 1. Puchner R, Janetschko R, Kaiser W, Linkesch \\ $M$, Steininger $M$, Tremetsberger R, Alkin A, \\ Machold K (2016) Efficacy and Outcome
}

of Rapid Access Rheumatology Consultation: An Office-based Pilot Cohort Study. J Rheumatol 43(6):1130-1135. doi: 10.3899/ jrheum. 151210

2. Puchner R, Edlinger M, Mur E, Eberl G, Herold M, Kufner P, Puchner A, Puchner SE, Redlich K, Alkin A, Machold K (2016) Interface Management between General Practitioners and Rheumatologists-Results of a Survey Defining a Concept for Future Joint Recommendations. PLoS One 11(1):e0146149. doi: 10.1371/journal.pone.0146149 (eCollection 2016)

3. Agca R, Heslinga SC et al (2016) Atheroslerotic cardiovascular disease in patients with chronic inflammatory joint disorders. Heart 102:790-795
4. Peters MJL, Symmons DPM, McCarey D et al (2010) EULAR evidence-based recommendations for cardiovascular risk management in patients with rheumatoid arthritis and other forms of inflammatory arthritis. Ann Rheum Dis 69:325-331

rheuma plus $2016 \cdot 15: 88-92$

DOI 10.1007/s12688-016-0096-9

Online publiziert: 24. Oktober 2016

(c) Springer-Verlag Wien 2016

\title{
Kompakte News vom ACR/ARHP Annual Meeting 2016 in Washington
}

Die Kongressberichterstattung Rheumatology TODAY informiert über die neuesten Studienergebnisse des ACR/ARHP Annual Meeting. 13 deutsche Rheumatologen besuchen in Washington relevante Vorträge und fassen die Forschungsdaten vor der Kamera zusammen. Die Inhalte stehen ab dem 14. November, 7 Uhr auf www.rheumatology-today.de als Videos mit Präsentationsfolien kostenlos zum Download zur Verfügung.

Die aktuellen Studienergebnisse und Highlights werden nach Schwerpunktthemen sortiert und von folgenden Referenten vorgestellt:

- PRO \& Burden of Disease: Dr. Rieke Alten, Schlosspark-Klinik, Universitätsmedizin Berlin

- Monotherapie als Option: Dr. Christopher Amberger, Rheumatologische Gemeinschaftspraxis Bad Neuenahr

- Vaskulitiden: Dr. Peer M. Aries, Rheumatologie im Struenseehaus, Hamburg

- Axiale Spondylarthritis: Dr. Xenofon Baraliakos, Rheumazentrum Ruhrgebiet Herne

- Psoriasis Arthritis: Dr. Frank Behrens, CIRI/Rheumatologie \& Fraunhofer TMP, Universitätsklinikum Goethe-Universität, Frankfurt a.M. munications gmbH
- Juvenile Idiopathische Arthritis: Prof. Dr. Gerd Horneff, Asklepios Kinderklinik, Sankt Augustin

- Komorbiditäten \& RA: Dr. Stefan Kleinert, Rheumatologische Schwerpunktpraxis, Erlangen

- RA - Aktuelle Therapieoptionen: Prof. Dr. Ulf Müller-Ladner, Kerckhoff-Klinik Bad Nauheim

- Biologica Safety: Prof. Dr. Andrea Rubbert-Roth, Uniklinik Köln

- Kollagenosen: Prof. Dr. Christof Specker, St. Josef Krankenhaus, Universitätsklinikum Essen

- Bildgebung: Prof. Dr. Johannes Strunk, Krankenhaus Porz am Rhein, Köln

- RA - Neue Targets und Biomarker: Prof. Dr. Torsten Witte, Medizinische Hochschule Hannover

„Unser Anliegen ist es, Rheumatologen ein kompetenter und zuverlässiger Partner in der Rheumatologie zu sein. Aus diesem Grund bieten wir mit Rheu- matology TODAY ein modernes und praxisrelevantes Weiterbildungstool, das Medizinern die neuesten Erkenntnisse in der Rheumatologieforschung in kompakter Form zugänglich macht. So können Rheumatologen ganzjährig von den neuen Forschungsdaten des Kongresses profitieren und dadurch zu einer verbesserten Versorgungssituation in Deutschland beitragen", so Dr. Bernd König, Direktor Marketing und Vertrieb, Chugai Pharma.

Alle Rheumatologie-Neuigkeiten stehen unter www.rheumatologytoday.de zur Verfügung. Der Zugang erfolgt über einen DocCheck-Account.

rheuma plus $2016 \cdot 15: 92$ DOI 10.1007/s12688-016-0094-y Online publiziert: 24. Oktober 2016 (c) Springer-Verlag Wien 2016 